\title{
Multiple drug-resistant Vibrio cholerae responsible for cholera outbreak among migrant domestic workers in Kerala, South India
}

\author{
Kiran Krishnaa, Abdulaziz Anas ${ }^{a^{*}}$, Sreelakshmi P.Ka ${ }^{a}$, Syamkumar Va, Jasmin Ca, Beena \\ Philomina $^{b}$ and Sobha Kurienc \\ a Regional Centre, CSIR - National Institute of Oceanography, Cochin, Kerala, 682018, India \\ b Microbiology department, Government Medical College, Calicut, Kerala, 673008, India \\ c Microbiology department, Government Medical College, Kottayam, Kerala, 686008, India \\ *Corresponding author - Anas Abdulaziz, Regional Centre, CSIR - National Institute of Oceanography, \\ Cochin, Kerala, 682018, India \\ Telephone: +91 484 2390814; Fax: +91 4842390618; Email: anas@nio.org \\ + Presented at the 1st International Electronic Conference on Microbiology, 2-30 November 2020; Available \\ online: https://ecm2020.sciforum.net/
}

Published: 2 November 2020

\begin{abstract}
Cholera outbreaks are prevalent in countries with low Human Development Index (HDI) where people have limited access to safe drinking water, sanitation and hygiene (WASH). Intriguingly, state of Kerala which records the highest HDI in India is endemic to cholera. We discuss the epidemiology of a cholera outbreak reported among migrant workers of Kerala in 2017. Virulence genes of Vibrio cholerae, tox R and ctx A, were detected in the river and ground water samples collected from the outbreaks sites which indicates need of enhanced awareness on WASH practices among migrant workers. The pathogenic Vibrio cholerae isolated from four patients in two districts had a similar DNA band pattern when analysed using repetitive extragenic palindromicPCR (BOX), which indicates their single clonal origin. The four isolates were serotyped as O1 Ogawa. These isolates were resistant to multiple antibiotics including the carbapenem like imipenem. Nevertheless, isolates of these pathogenic bacteria were susceptible to tetracycline identical to clinical isolates of Vibrio cholera reported earlier from this region. Current study highlights the importance of generating awareness on WASH protocols among migrant workers to prevent the outbreaks and considers community-based data as socioeconomic variable to predict the incidence of cholera.
\end{abstract}

Keywords: Cholera; antibiotic resistance; epidemiology; outbreak; migration.

\section{Introduction}

Cholera, an acute diarrhoeal disease caused by water associated pathogen Vibrio cholerae, infects a major share of world population exposed to poor water, sanitation and hygiene (WASH) facilities [1]. Many developed countries could prevent the outbreak of disease following the WASH procedure, which was first linked to the epidemiology of this disease by John Snow in 1854 [2]. World Health Organization (WHO) has proposed several strategies to eliminate disease transmission by 2030, among which the important one is WASH [3]. However, several populations in Asia and Africa are deprived of access to safe drinking water and sanitation due to low socio-economic conditions represented by low Human Development Index (HDI) [4] and pollution. The Indian subcontinent has been the homeland of Asiatic cholera as major pandemics of this disease originated from this region and disseminated along with human migration and trade to different regions of the world [5]. According to the infectious disease surveillance programme (IDSP), 13 states of India are endemic to 
cholera [6]. Intriguingly, this list includes state of Kerala which records the highest HDI in the country [7] where an outbreak was reported among migrant workers in 2017. Among the four people diagnosed with cholera, one succumbed to death.

Though the first line treatment for cholera is oral rehydration therapy, antibiotics are also prescribed to reduce the severity of its symptoms such as water loss as well as shedding of the pathogen from the patient. Epidemiological studies revealed that chemoprophylaxis contributed substantially to the emergence of new clones of Vibrio cholerae often displaying reduced susceptibility to multiple antibiotics forming a major impediment in treatment of this disease [8]. The emergence of multiple antibiotic resistant cholera pathogens and reports on climate associated changes, such as global warming and flood associated with torrential rains, favouring the incidence of cholera are alarming. It is important to characterize the isolates responsible for each outbreak to understand the evolution of Vibrio cholerae and disease pattern. In this regard, our study deals with the epidemiological investigations of cholera outbreak among migrant workers as well as the antibiotic resistance profile of Vibrio cholerae isolated from infected individuals.

\section{Materials and Methods}

The water samples $(25 \mathrm{ml})$ collected from the river and ground water source in the affected areas were enriched in alkaline peptone broth $(225 \mathrm{ml})$ for $24 \mathrm{~h}$ at room temperature (Fig. 1). Subsequently, an aliquot $(1.5 \mathrm{ml})$ of the enriched broth was transferred into a microcentrifuge tube and the cells were separated by centrifugation at $8000 \mathrm{rpm}$ for $10 \mathrm{~min}$. The cells were suspended in $100 \mu \mathrm{L}$ of TE buffer ( $\mathrm{pH}-8)$ and lysed by keeping in a boiling water bath for 10 minutes followed by cold shock. Cell lysate thus prepared was further centrifuged at $7000 \mathrm{rpm}$ for 10 minutes at $4{ }^{\circ} \mathrm{C}$ and supernatant was used as template DNA for PCR. Alongside, the clinical isolates of $V$. cholerae were cultivated in Luria Bertani (LB) broth and the genomic DNA was extracted by following CTAB-NaCl method [9]. DNA samples were further analysed for the presence of virulence genes, tox $\mathrm{R}$ and ctx A, of Vibrio cholerae using primer sets specific for tox R ( F - 5' CCTTCGATCCCCTAAGCAATAC 3'; R - 5' AGGTTAGCAACGATGCGTAAG 3') and ctx A (F - 5' AAC TCA GAC GGG ATT TGT TAG GC 3'; $\mathrm{R}$ - 5' TCT CTG TAG CCC CTA TTA CGA TGT 3') [10]. BOX-PCR was also used for phylogenic grouping of clinical isolates of $V$. cholerae from different sources. Here the palindromic regions in the DNA of each organism was amplified using BOX A1R primer 5'CTACGGCAAGGCGACGCTGACG-3' [11].

Serotyping of the clinical isolates of $V$. cholerae were determined as agglutination over glass slides with polyvalent $\mathrm{O} 1$ antiserum, monovalent serotypes of Inaba, Ogawa and antiserum of O 139 (Denka Seiken, Tokyo, Japan). The clinical isolates of $V$. cholerae were also screened for their antimicrobial susceptibility using the Kirby-Bauer disk diffusion method on Mueller-Hinton Agar. Isolates were tested against 15 antimicrobial drugs as follows : ampicillin $(25 \mu \mathrm{g})$, tetracycline $(30 \mu \mathrm{g})$, erythromycin $(15 \mu \mathrm{g})$, gentamicin $(30 \mu \mathrm{g})$, trimethoprim $(30 \mu \mathrm{g})$, cefalexin $(30 \mu \mathrm{g})$, cefepime $(30 \mu \mathrm{g})$, cefoxitin $(30 \mu \mathrm{g})$, ceftriaxone $(30 \mu \mathrm{g})$, chloramphenicol $(30 \mu \mathrm{g})$, gatifloxacin $(5 \mu \mathrm{g})$, imipenem $(10 \mu \mathrm{g})$, moxifloxacin $(5 \mu \mathrm{g})$, nalidixic acid $(30 \mu \mathrm{g})$ and norfloxacin $(10 \mu \mathrm{g})$ (all Hi media Labs, Mumbai, India). The isolates were classified into resistant or sensitive based on zone of inhibition interpreted as per manufacturer's instructions. 


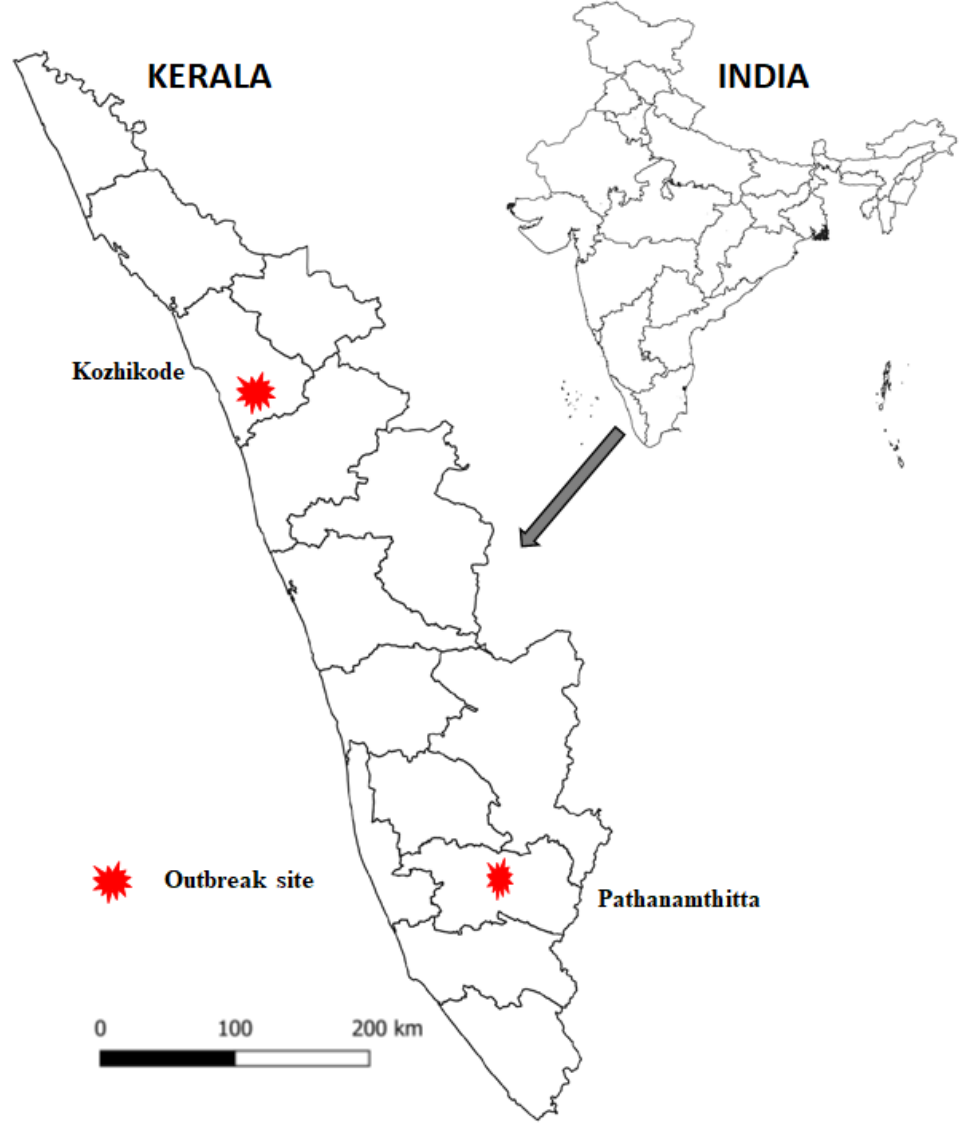

Figure 1. Map showing districts wherein cholera outbreak was reported in Kerala.

\section{Results \& Discussion}

Water samples collected from the river and ground water sources in the cholera infected regions were found harbouring virulence genes of $V$. cholerae. Disease incidence had reported only among migrant workers and were not transmitted to local population. This could have happened because of two reasons 1) they might have carried the disease from their home town or 2) must have consumed the contaminated water without boiling or not followed the WASH protocols. The cholera outbreaks have been reported among migrant workers and refugees in several areas across the globe and have been attributed to overcrowding, limited access to safe drinking water and poor sanitation and hygiene practices [12-14]. The living conditions of migrant labourers are poor in Kerala with several people sharing single rooms with less provision of hygiene sanitation [15]. The high population density ( 860 persons $/ \mathrm{km}^{2}$ according to census 2011 data), less availability of safe drinking water (Fig. 2), prolonged monsoon and prevalence of $V$. cholerae in the surface water makes the region endemic to cholera. The environmental monitoring studies conducted in this area also confirmed the prevalence of $V$. cholerae in the aquatic environments thorough out the year [16]. Water associated diseases are considered as one of the major reasons for reduced man working days in the region [17]. However, the death due to cholera are less in Kerala compared to other endemic states in India and is attributed to its high HDI (Fig. 2) achieved through various public health measures taken in the past few decades such as vaccination campaigns, sanitation, water supply and personal environmental hygiene [18]. 


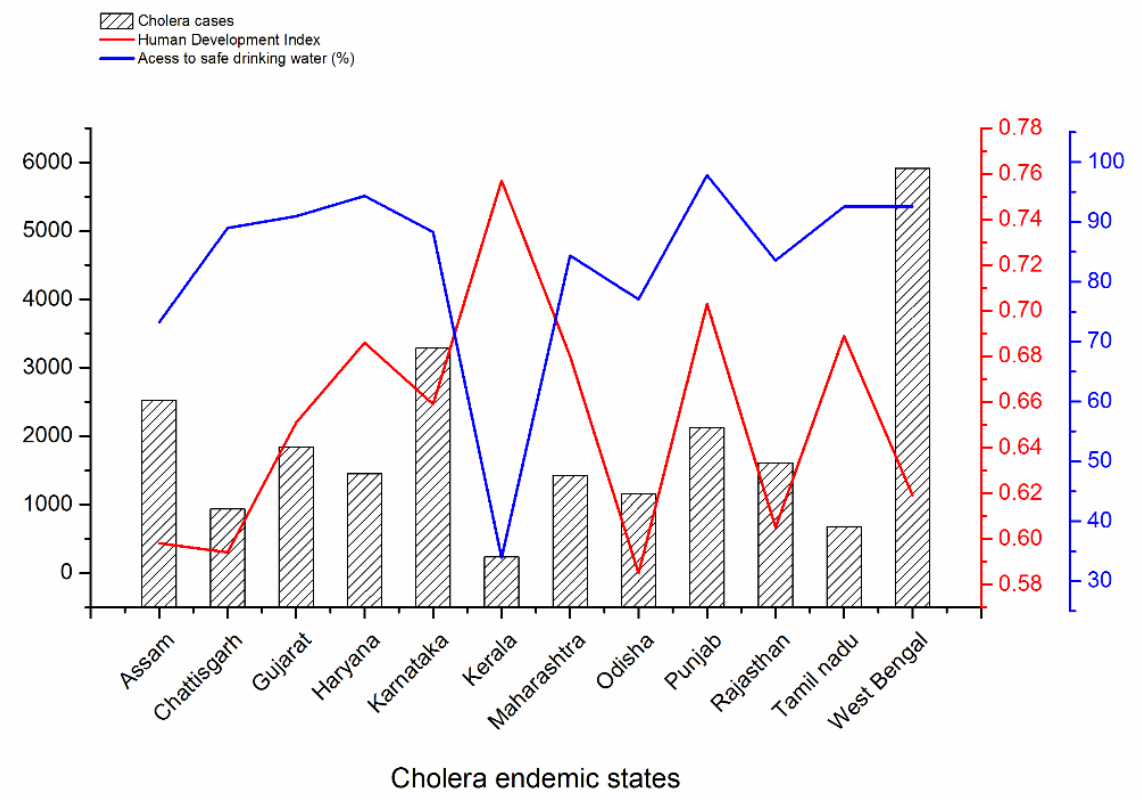

Figure 2. Cumulative cholera case (2010-2015) in 12 endemic states of India plotted against their respective Human development Index and \% of population access to safe drinking water.

Further analysis showed that all the four clinical strains of $V$. cholerae isolated from Kozhikode and Pathanamthitta were pathogenic harbouring tox $\mathrm{R}$ and ctx A genes and serological analysis grouped them as O1, Ogawa. The pathogenicity of $V$. cholerae is controlled by the synergistic effect of different genes initiated by the transcriptional activator tox $\mathrm{R}$ which initiates a cascade of gene expressions leading to the production of cholerae toxin encoded by the ctx gene. The BOX PCR pattern showed that the $V$. cholerae isolated from infected persons in distant regions (Kozhikode and Pathanamthitta) were same clone with similar band patterns (Fig. 3).

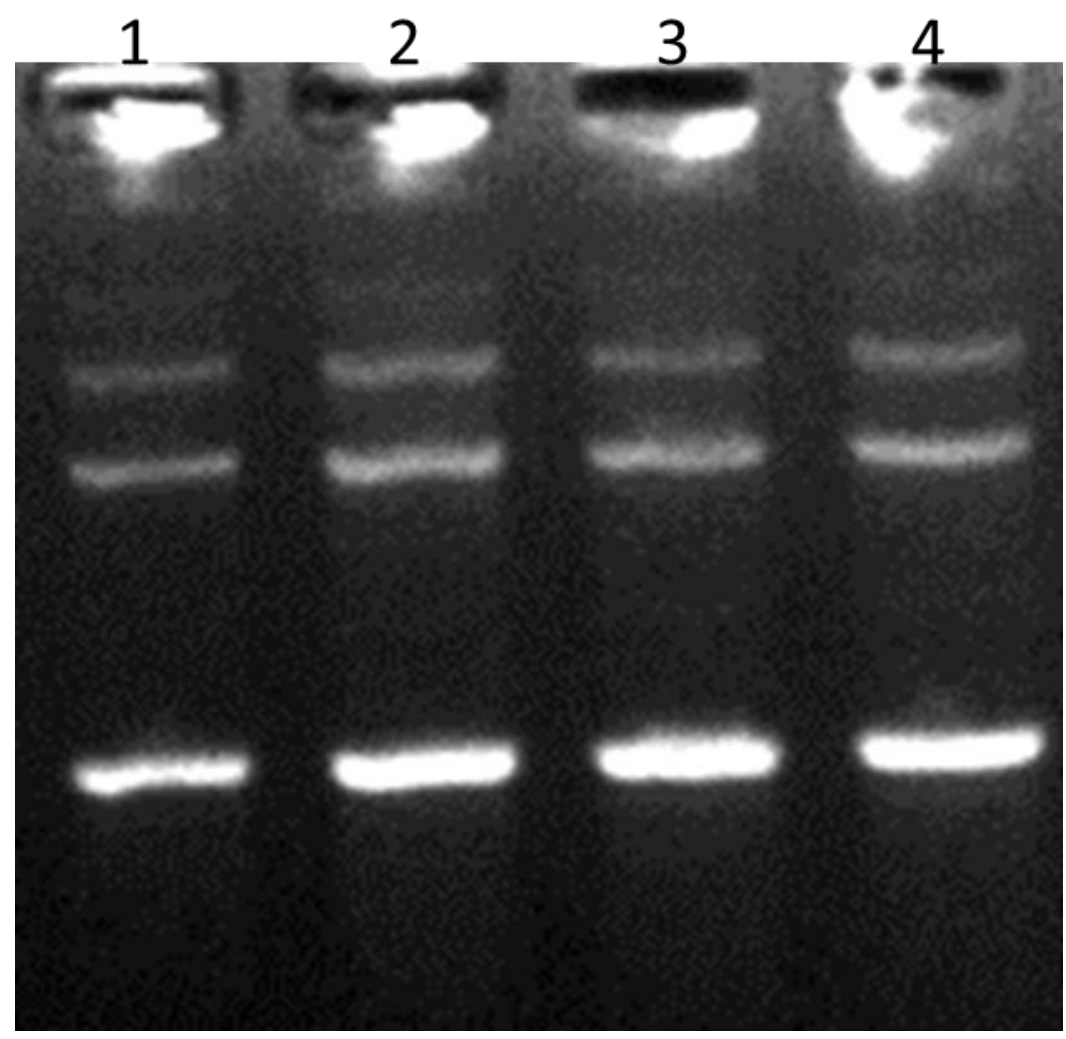


Figure 3. Agarose gel image of the box PCR patterns of $V$. cholerae isolated from Pathanamthitta (lane 1) and Kozhikode (lane 2, 3 and 4).

All four clinical isolates of $V$. cholerae were resistance to multiple antibiotics with surprisingly more or less similar susceptibility profile (Table 1).

Table 1. Antibiotic resistance profile of clinical $V$. cholerae isolates.

\begin{tabular}{cccccc}
\hline \multirow{2}{*}{ Chemical class } & \multirow{2}{*}{ Antibiotics } & \multicolumn{4}{c}{ Source of V.cholerae } \\
\cline { 2 - 6 } & & Pathanamthitta & Kozhikode & Kozhikode & Kozhikode \\
\hline Penicillin & Ampicillin & $\mathrm{R}$ & $\mathrm{R}$ & $\mathrm{R}$ & $\mathrm{R}$ \\
\hline \multirow{3}{*}{ Cephalosporins } & Cephalexin & $\mathrm{R}$ & $\mathrm{R}$ & $\mathrm{R}$ & $\mathrm{R}$ \\
\cline { 2 - 6 } & Cefoxitin & $\mathrm{R}$ & $\mathrm{R}$ & $\mathrm{R}$ & $\mathrm{R}$ \\
\cline { 2 - 6 } & Ceftriaxone & $\mathrm{R}$ & $\mathrm{R}$ & $\mathrm{R}$ & $\mathrm{R}$ \\
\cline { 2 - 6 } & Cefapime & $\mathrm{R}$ & $\mathrm{R}$ & $\mathrm{R}$ & $\mathrm{R}$ \\
\hline Carbapenem & Imipenem & $\mathrm{R}$ & $\mathrm{R}$ & $\mathrm{R}$ & $\mathrm{R}$ \\
\hline Tetracycline & Tetracycline & $\mathrm{S}$ & $\mathrm{S}$ & $\mathrm{S}$ & $\mathrm{S}$ \\
\hline Aminoglycoside & Gentamicin & $\mathrm{R}$ & $\mathrm{R}$ & $\mathrm{S}$ & $\mathrm{R}$ \\
\hline Macrolides & Erythromycin & $\mathrm{R}$ & $\mathrm{R}$ & $\mathrm{R}$ & $\mathrm{R}$ \\
\hline Chloramphenicol & Chloramphenicol & $\mathrm{R}$ & $\mathrm{R}$ & $\mathrm{R}$ & $\mathrm{R}$ \\
\hline \multirow{3}{*}{ Fluoroquinolones } & Nalidixic acid & $\mathrm{R}$ & $\mathrm{R}$ & $\mathrm{R}$ & $\mathrm{R}$ \\
\cline { 2 - 6 } & Norfloxacin & $\mathrm{R}$ & $\mathrm{R}$ & $\mathrm{R}$ & $\mathrm{R}$ \\
\cline { 2 - 6 } & Gatifloxacin & $\mathrm{R}$ & $\mathrm{R}$ & $\mathrm{S}$ & $\mathrm{R}$ \\
\cline { 2 - 6 } & Moxifloxacin & $\mathrm{R}$ & $\mathrm{R}$ & $\mathrm{S}$ & $\mathrm{R}$ \\
\hline Sulfonamide & Trimethoprim & $\mathrm{R}$ & $\mathrm{R}$ & $\mathrm{R}$ & $\mathrm{R}$ \\
\hline
\end{tabular}

All isolates were susceptible to tetracycline, one of the mainstream drugs used worldwide for the treatment of cholera in adults. The resistance of VC against this antibiotic is often reported sporadic from Indian subcontinent. $V$. cholerae isolated from a previous outbreak in Kerala do confirm the same [19]. The consistent susceptibility of VC isolates towards tetracycline should be welcomed as indicates that this antibiotic is still effective against this pathogen found in these regions. Alarmingly, all the isolates were resistant to, cell wall synthesis inhibiting antibiotics tested belonging to, penicillins (ampicillin) as well as first to fourth generations of cephalosporins (cephalexin, cefoxitin, ceftriaxone and cefapime) and carbapenem (imipenem). Reports of VC strains attaining resistance against first generation cephalosporin like cephalexin, dates back as early as 2000. Whereas the resistance of a $V$. cholerae against third generation cephalosporin (ceftrioxine) was earlier reported from south India and was attributed to the activity of either extended spectrum of $\beta$-lactamase (ESBL) or Amp C $\beta$-lactamase [20]. The growth of cephalosporin resistant microorganism is often inhibited by usage of antibiotics belonging carbapenem family. However, in this case, all the isolates of $V$. cholerae showed resistance to imipenem, the carbapenem class of antibiotic, making these isolates further similar to aforementioned strain isolated from south India. Studies in future to understand the emergence of cephalosporin resistance among $V$. cholerae should be appreciated, as these antibiotics are not generally prescribed as therapeutic agents for cholera.

All four isolates were also resistant against DNA synthesis inhibitor antibiotic belong to the class of first and second generation quinolones (Nalidixic and norfloxacin) tested. Long term studies conducted in National Institute of Cholera and Enteric Disease (NICED) reveal that resistance of VC isolates observed against nalidixic and norfloxacin was acquired as early as 1994 and 2000 respectively [21]. Except one isolate from Kozhikode, remaining all were resistant against latest generation fluoroquinolones tested viz. Gatifloxacin and Moxifloxacin. The resistance of VC isolates 
against gentamicin is of concern as they are reported seldom globally. Out of 25 clinical isolates of VC from a previous outbreak in Kerala, only one emerged resistant to this antibiotic $(10 \mathrm{mcg})$ that too at a concentration tested threefold lower than our current study [19]. Hence the current results should be noted with caution. Most strains isolated from India were resistance to trimethoprim whereas, susceptibility to chloramphenicol and erythromycin though reported, were low in incidence.

Sustainable availability of low skilled jobs with high wage structure (Rs $\sim 600 /$ day, double the national average) and other welfare schemes in Kerala is an attraction to migrant workers from other states, which is indicated by the increase in their number from 2.5 in 2013 to 4.0 million in 2017 [22]. Poor WASH practice, less concern about sanitation and hygiene prevailed in the residential areas of these population may trigger the cholera outbreaks in a state like Kerala, where the $V$. cholerae are prevalent in the environment. Our study indicates the need for providing decent accommodation and sanitation facilities to them and conduct awareness programs on WASH procedure to prevent the cholera outbreaks in future. Also, is important to consider community based data as a socioeconomic variable to predict cholera outbreaks in the country.

Acknowledgments: The authors thank the Director, CSIR-National Institute of Oceanography, Goa, the Director, ICMAM-PD (MoES), Chennai and Scientist-in-Charge CSIR-NIO regional centre, Kochi, for extending all required support. This work was done using MMRF facility and is duly acknowledged. The authors declare they have no conflict of interest.

This is a CSIR-NIO contribution \# xxx.

\section{References}

1. Taylor DL, Kahawita TM, Cairncross S, Ensink JHJ (2015) The Impact of Water, Sanitation and Hygiene Interventions to Control Cholera: A Systematic Review. PLOS ONE 10: e0135676. https://doi.org/10.1371/journal.pone.0135676

2. Snow J (1855) On the Mode of Communication of Cholera. John Churchill

3. World Health Organization (2019) WHO global water, sanitation and hygiene: annual report 2018.

4. Ackers M-L, Quick RE, Drasbek CJ, et al (1998) Are there national risk factors for epidemic cholera? The correlation between socioeconomic and demographic indices and cholera incidence in Latin America. International Journal of Epidemiology 27:330-334

5. Colwell RR (1996) Global climate and infectious disease: the cholera paradigm. Science 274:2025-2031

6. Ali M, Sen Gupta S, Arora N, et al (2017) Identification of burden hotspots and risk factors for cholera in India: An observational study. PLoS ONE 12: e0183100. https://doi.org/10.1371/journal.pone.0183100

7. Sub-national HDI - Subnational HDI - Global Data Lab. https://globaldatalab.org/shdi/shdi/IND/?interpolation=0\&extrapolation=0\&nearest_real=0\&years=2015. Accessed 31 March 2020.

8. Kitaoka M, Miyata ST, Unterweger D, Pukatzki S (2011) Antibiotic resistance mechanisms of Vibrio cholerae. Journal of medical microbiology 60:397-407

9. Wilson K (2001) Preparation of Genomic DNA from Bacteria. Current Protocols in Molecular Biology 56:2.4.1-2.4.5. https://doi.org/10.1002/0471142727.mb0204s56

10. Sneha KG, Anas A, Jayalakshmy KV, et al (2016) Distribution of multiple antibiotic resistant Vibrio spp across Palk Bay. Regional Studies in Marine Science 3:242-250

11. Zhu L, Xu H, Zhang Y, et al (2014) BOX-PCR and PCR-DGGE analysis for bacterial diversity of a naturally fermented functional food (Enzyme®). Food Bioscience 5:115-122

12. Md WS, Md CN, Mph PP (2008) An Outbreak of Cholera among Migrants Living in a Thai-Myanmar Border Area. Journal of Medical Association Thailand 91:1433-1440

13. Siddique AK (1994) Cholera epidemic among Rwandan refugees: experience of ICDDR,B in Goma, Zaire. Glimpse 16:3-4

14. Naficy A, Rao MR, Paquet C, Antona D, Sorkin A, Clemens JD (1998) Treatment and Vaccination Strategies to Control Cholera in Sub-Saharan Refugee Settings: A Cost-effectiveness Analysis. JAMA 279:521-525

15. Saikia D (2015) Migrant Workers in Kerala: A Study on Their Socio-Economic Conditions. Journal of Economic and Social Development 11:29-43. 
16. Krishna K, Veettil VP, Anas A, Nair S (2020) Hydrological regulation of Vibrio dynamics in a tropical monsoonal estuary: a classif ication and regression tree approach. Environ Sci Pollut Res. https://doi.org/10.1007/s11356-020-10486-9

17. Mani SK, Kanjur R, Raj BS, Surekha IS, Singh ISB, Reed RH Solar disinfection as a management strategy for drinking water treatment in rural households \& in natural disasters. https://www.researchgate.net/publication/257840643_Solar_disinfection_as_a_management_strategy_for_ drinking_water_treatment_in_rural_households_in_natural_disasters. Accessed 24 Aug 2020

18. Panikar PGK (1975) Fall in Mortality Rates in Kerala: An Explanatory Hypothesis. Economic and Political Weekly 10:1811-1818

19. Sabeena F, Thirivikramji G, Radhakutty G, et al (2001) In vitro susceptibility of Vibrio cholerae O1 biotype El Tor strains associated with an outbreak of cholera in Kerala, Southern India. Journal of antimicrobial chemotherapy 47:361-362

20. Mandal J, Sangeetha V, Ganesan V, et al (2012) Third-generation cephalosporin-resistant Vibrio cholerae, India. Emerging infectious diseases 18:1326-1328

21. Garg P, Sinha S, Chakraborty R, et al (2001) Emergence of fluoroquinolone-resistant strains of Vibrio cholerae O1 biotype El Tor among hospitalized patients with cholera in Calcutta, India. Antimicrobial agents and chemotherapy 45:1605-1606

22. Kumar NA (2011) Vulnerability of migrants and responsiveness of the state: The case of unskilled migrant workers in Kerala, India. (CSES Working Paper Series No.26) Centre for Soci-Economic and Environmental Studies, Kochin

Publisher's Note: MDPI stays neutral with regard to jurisdictional claims in published maps and institutional affiliations.

(C) 2020 by the authors; licensee MDPI, Basel, Switzerland. This article is an open access article distributed under the terms and conditions of the Creative Commons Attribution (CC-BY) license (http://creativecommons.org/licenses/by/4.0/). 